\section{P079 USING THEORY AND EVIDENCE TO OPTIMISE AN ACCELERATED PARTNER THERAPY INTERVENTION IN A CHLAMYDIA PARTNER NOTIFICATION TRIAL}

${ }^{1}$ Paul Flowers*, ${ }^{2}$ Maria Pothoulaki, ${ }^{3}$ Melvina Owusu, ${ }^{2}$ Gaby Vojt, ${ }^{3}$ Fiona Mapp, ${ }^{4}$ Catherine Mercer, ${ }^{5}$ Jackie Cassell, ${ }^{3}$ John Saunders, ${ }^{4}$ Sonali Wayal, ${ }^{6}$ Merle Symonds, ${ }^{7}$ Rak Nandwani, ${ }^{3}$ Alison Howarth, ${ }^{6}$ Alex Comer -Schwartz, ${ }^{8}$ S Brice, ${ }^{9}$ Claudia Estcourt. ${ }^{1}$ University of Glasgow, MRC/CSO Social and Public Health Sciences Unit, Glasgow, UKi ${ }^{2}$ Glasgow Caledonian University, Glasgow, UK; ${ }^{3} U C L$, London, UK; ${ }^{4}$ University College London, Institute for Global Health, London, UK; ${ }^{5}$ Brighton and Sussex Medical School, Primary Care and Public Health, Brighton and Hove, UK; ${ }^{6}$ Barts Health NHS Trust, London, UK; ${ }^{7}$ NGS Greater Glasgow and Clyde, Glasgow, UK; ${ }^{8}$ NHS Barts, London, UK; ${ }^{9}$ Glasgow Caledonian University, School of Health and Life Sciences, Glasgow, UK

\subsection{6/sextrans-2019-sti.276}

Background Accelerated Partner Therapy (APT) is a method of partner notification (PN) which includes remote assessment, self-sampling and treatment of the sex partner(s) of a person diagnosed with STIs. Using the Behaviour Change Wheel approach, we sought to understand, and then systematically moderate, the psychological and behavioural challenges involved in delivering and receiving APT as part of LUSTRUM, a UK-based chlamydia PN trial.

Methods We conducted 11 focus groups with patients $(n=30)$ and the public $(n=26)$, and five focus groups with healthcare professionals $(n=30)$ involved in PN. Initial thematic analysis explored the barriers and facilitators to each essential and sequential step in APT, from the perspective of each role in this interpersonal, relay intervention. Further analysis specified a series of theoretically based, and evidence-informed approaches that can be used to methodically reduce the various barriers to uptake and implementation of APT

Results Active, intervention components that can enhance staff delivery, index patient and sex partner uptake of APT included providing: clearer information for all about the overall sequential steps involved in APT (highlighting shared roles and responsibilities); a stronger focus upon what healthcare professionals, index patients and their sex partners should do in relation to each step of APT, including written checklists for staff and index patients; essential messages supporting PN and self-management of sex partners engaging in APT; pertinent information about why to engage in APT (in relation to considering key consequences).

Conclusion This is the first study to provide evidence-based and theoretically informed approaches to enhance contemporary $\mathrm{PN}$. These findings informed the development of an intervention manual, training resources for staff and online video materials. Together, these materials detail, in highly specific and replicable ways, the optimal methods to reduce barriers to implementation of APT and utilise factors that facilitate implementation thereby potentially improving PN outcomes for chlamydia.

Disclosure No significant relationships.

\section{P080 INVESTIGATING THE EFFECTS OF ACCELERATED PARTNER THERAPY ON CHLAMYDIA TRANSMISSION IN BRITAIN: A MATHEMATICAL MODELLING STUDY}

${ }^{1}$ Christian Althaus*, ${ }^{2}$ Catherine Mercer, ${ }^{3}$ Jackie Cassell, ${ }^{4}$ Claudia Estcourt, ${ }^{5}$ Nicola Low. ${ }^{1}$ University of Bern, Institute of Social and Preventive Medicine, Bern, Switzerland; ${ }^{2}$ University College London, Institute for Global Health, London, UK; ${ }^{3}$ Brighton and Sussex Medical School, Primary Care and Public Health, Brighton and Hove, UK; ${ }^{4}$ Glasgow Caledonian University, School of Health and Life Sciences, Glasgow, UK; ${ }^{5}$ University of Bern, Institute of Social and Preventive Medicine (ISPM), Bern, Switzerland

\subsection{6/sextrans-2019-sti.277}

Background Understanding the effects of partner notification (PN) on chlamydia transmission is critical for implementing optimal control strategies. Accelerated partner therapy (APT) aims to reduce the time to partner treatment and increase the proportion of partners treated. As part of LUSTRUM, a PN trial in the UK, our objective was to study the effects of APT interventions on partner treatment and chlamydia transmission using a mathematical model.

Methods We developed a deterministic, population-based chlamydia transmission model including the process of PN. We considered a heterosexual population aged $16-34$ years and calibrated the model to sexual behaviour and chlamydia prevalence data reported by 3,671 participants in Britain's third National Survey of Sexual Attitudes and Lifestyles (Natsal-3, 2010-2012) using Approximate Bayesian Computation (ABC). We investigated the potential effects of APT on chlamydia transmission by reducing the time to partner treatment and increasing the proportion of treated partners compared to standard PN.

Results The median prevalence of chlamydia in the model was $1.8 \%$ (95\% credible interval (CrI): $1.6 \%-2.6 \%)$ in women and 1.7\% (95\% CrI: 1.1\%-2.1\%) in men. Overall, chlamydia-positivity in partners of index cases was 55\% (95\% CrI: 31\%-79\%), and higher in partners of symptomatic index cases (76\%) than asymptomatic index cases (33\%). Reducing the time to partner treatment without achieving higher proportions of partners treated had only small effects on reducing chlamydia prevalence. In contrast, the model predicts that a potential increase in the proportion of partners treated from current levels in the UK (36\%, 95\% CrI: $13 \%-96 \%$ ) by $25 \%$ would reduce chlamydia prevalence by $14 \%$ (95\% CrI: $3 \%-40 \%)$ in women and by 14\% (95\% CrI: 3\%-39\%) in men within 5 years.

Conclusion Our results suggest that APT, through an increase in the proportion of partners treated, would be an effective method to reduce ongoing chlamydia transmission in the UK. Disclosure No significant relationships. 